\title{
Procedimiento PaRa la eVALUACIÓN \\ DE LOS FACTORES DE RIESGO LABORAL \\ Y SU INCIDENCIA EN EL DESEMPEÑO \\ LABORAL EN USUARIOS DE PANTALLAS \\ DE VISUALIZACIÓN DE DATOS (PVD)
}

GRETHER REAL

https://orcid.org/0000-0003-4792-6217

Liliana Cedeño

https://orcid.org/0000-0003-4298-4557

Universidad Técnica de Manabí, Ecuador

Recibido: 7 de agosto del 2020 / Aprobado: 18 de agosto del 2020

doi: https://doi.org/10.26439/ing.ind2020.n039.4913

RESUMEN: El objetivo de esta investigación es proponer un procedimiento que permita evaluar los factores de riesgo laboral y su incidencia en el desempeño laboral en usuarios de PVD. Los métodos de investigación considerados fueron el exploratorio, descriptivo, trabajo de campo y análisis-síntesis. El procedimiento consta de cuatro etapas: diagnóstico de la situación actual, evaluación de riesgo, incidencia de los riesgos en el desempeño laboral y control de riesgo. Su principal aporte radicó en la compilación, selección, propuesta y explicación de las herramientas que permiten el estudio en diferentes trabajadores que sus actividades se centran en el uso de PVD.

Palabras clave: factor de riesgo laboral / desempeño laboral / usuario de PVD / ergonomía / bienestar laboral

*Correo electrónico: greal@utm.edu.ec, gretherreal@gmail.com, Icedeno1@hotmail.com 


\title{
A PROCEDURE FOR THE EVALUATION OF OCCUPATIONAL RISK FACTORS AND THEIR IMPACT ON WORK PERFORMANCE AMONG USERS OF DATA DISPLAY SCREENS (DDSS)
}

\begin{abstract}
The objective of this research is to propose a procedure that allows the evaluation of occupational risk factors and their impact on the work performance of DDS users. The research considered exploratory, descriptive, fieldwork and synthesis analysis methods. The procedure consisted of four stages: diagnosis of the current situation, risk assessment, incidence of risks in work performance, and risk control. Its main contribution lies in the compilation, selection, proposal and explanation of the tools that allow studying different workers whose activities are focused on the use of DDS.
\end{abstract}

Keywords: occupational risk factor / job performance / DDS user / ergonomics / work well-being 


\section{INTRODUCCIÓN}

El presente estudio tiene el propósito de realizar una propuesta de un procedimiento que permita la valoración de los factores de riesgo que están presentes en usuarios de PVD y su incidencia en el desempeño laboral, definiendo las herramientas o metodologías que permitan identificar las brechas de mejoras en los puestos y con ello, la garantía de la realización del trabajo decente, sin riesgo, y sin afectar el desempeño laboral.

El trabajo es una actividad que permite satisfacer una necesidad y conseguir determinados objetivos; si se trabaja dentro de una organización o empresa, esta debe tener la habilidad de conseguir que los trabajadores quieran hacer el trabajo, y además, que lo hagan bien (Carballé Piñón, 2015). Para Durany Puig (2019), trabajar es una actividad realizada por todas las personas en algún momento de su vida; sin embargo, pocos reflexionan sobre la manera en la que el trabajo puede llegar a afectar la salud.

El talento humano es el que hace diferentes, competitivos y añade valor a los procesos productivos, ya sean de servicio o de producción. Una de las estrategias empresariales que permite alcanzar altos índices de productividad y eficiencia es el fortalecimiento del compromiso del trabajador con su organización, desvirtuando cualquier conflicto que deteriore su estadía en la empresa (Ccollana-Salazar, 2017). Para ello, se hace necesario mantener un clima laboral excelente donde todos estén a gusto sabiendo que sus expectativas se cubren y se incrementa su nivel de vida, apoyando sus objetivos personales y organizacionales (Bravo y Salazar, 2017).

Cuesta y Valencia (2018) se refieren a la evolución de la gestión del personal como estrategia competitiva, que tiene su auge a partir de la década de 1980. En este proceso han sido utilizados varios términos, tales como como: Gestión de Recursos Humanos (GRH), Gestión del Talento Humano (GTH), Gestión de Personal (GP), Gestión Humana $(\mathrm{GH})$, entre muchos otros. La esencia radica en ubicar al ser humano como eje central de los procesos productivos o servicios, atendiendo de manera integral todas sus necesidades biológicas, psicológicas y sociales.

La gestión de riesgos laborales tiene una relación con los resultados del desempeño laboral, permitiendo que el trabajador realice sus actividades en ambientes decentes y su desempeño garantice una calidad adecuada en el trabajo. El cuidado y preservación de la salud en los trabajadores es un factor clave para el incremento de la productividad, la sostenibilidad del trabajo realizado y el bienestar de todos sus empleados (Real et al., 2019).

Hoy en día, en la mayoría de los puestos se debe hacer uso de computadoras o Pantallas de Visualización de Datos (PVD) que ayudan a los trabajadores a desempeñar sus labores con mayor eficacia y productividad. Sin embargo, estas nuevas exigencias en la vida laboral engendran nuevos riesgos que tienen una incidencia directa en la salud y 
el bienestar y son una causa importante de los índices de ausentismo y de muchas enfermedades generadas por el estrés y las formas y exigencias de organización del trabajo, cada vez más comunes en el personal que labora en las oficinas o con el uso mantenido de las tecnologías de la información y las comunicaciones.

Uno de los principales riesgos en el uso de PVD son las afectaciones músculoesqueléticas en estos usuarios. Los trastornos músculo-esqueléticos (TME) son lesiones del aparato locomotor con un origen laboral. Afectan principalmente la espalda, cuello, hombros y extremidades superiores (Paredes y Vásquez, 2018).

Dentro de los factores de riesgo que inciden en los TME se encuentran las condiciones de trabajo, el entorno entre el usuario y las PVD, el diseño físico del puesto, los movimientos repetitivos, las posturas forzadas, el régimen de trabajo, el tiempo de descanso y los factores psicosociales, entre otros. Todos estos componentes tienen una incidencia directa en el desempeño de los trabajadores, afectando su eficiencia, rendimiento y productividad.

En la actualidad, las organizaciones se hallan en una incesante necesidad de mejorar su desempeño laboral con el fin de alcanzar la competencia en el mercado (Carmona y Rosas, 2017). Sobre el desempeño laboral existen variadas definiciones, que relacionan tanto las exigencias técnicas, productivas y los resultados del servicios que la empresa espera de él (López, Camal y Galicia, 2018).

Conocer las necesidades reales o sentidas que influyen en el desempeño laboral es una prioridad que permite ejecutar acciones tendientes a ofrecer a los trabajadores aquellos elementos que contribuyan a lograr la satisfacción en el desempeño de sus funciones, lo cual se verá reflejado en la productividad y el ambiente de trabajo (Arboleda y Cardona, 2018).

La prevención de riesgos laborales está amparada en la normativa legal, materializada en las leyes, acuerdos, decretos y reglamentos, que justifican la necesidad de realizar estudios que promuevan el trabajo decente y sin riesgos.

En el ámbito internacional, en marzo del 2018 se publica la ISO 45001 con la propuesta de un modelo de Gestión Efectiva de Seguridad y Salud en el Trabajo, la cual es una norma internacional certificable a cualquier empresa, y presenta una estructura de alto nivel que permite la integración con los otros sistemas de gestión en el ámbito empresarial.

En el Ecuador, en la sección tercera, en su artículo 326 se plantea que: "[...] Toda persona tendrá derecho a desarrollar sus labores en un ambiente adecuado y propicio, que garantice su salud, integridad, seguridad, higiene y bienestar" (Asamblea Nacional de la República del Ecuador, 2018). 
En el plano metodológico, se tienen como referente un conjunto de metodologías o métodos que permiten la gestión de riesgos en diferentes sectores o puestos laborales. Autores como Aulestiarte y Villanueva (2013) realizan una comparación de muchos de ellos, haciendo referencia a las características principales de cada uno.

Se presentan los métodos de evaluación global, incluidos LEST, Renault y ERGOS, que consideran diferentes factores de riesgo y su manera de evaluarlos. Adicionalmente, se muestran otros métodos de evaluación de algún factor de riesgo específico, como INSHT y NIOSH para la evaluación de la manipulación manual de carga; JSI para la evaluación de los movimientos repetitivos; REBA, OWAS y RULA, para la evaluación de la carga postural considerando las posturas forzadas y muchos otros métodos que consideran en su evaluación diferentes factores de riesgo (Buitrago, 2016).

En el año 2011 se dio a conocer el método ROSA, con el objetivo de hacer una evaluación del riesgo por el uso de las PVD. El método ROSA calcula la desviación existente entre las características del puesto evaluado y las de un puesto de oficina de características ideales (Diego-Mas, 2020). Otros autores, como Palacio y otros (2017) han usado otros métodos, como el Cuestionario de desórdenes músculo esqueléticos propuesto por Kuorinka en 1987 y el Cuestionario de autopercepción de la postura frente a una pantalla de visualización de datos, que permiten dar respuesta a diferentes hipótesis en este campo.

Aunque hay muchísimos métodos para la evaluación de los factores de riesgo, existen dificultades en la selección adecuada del método que permite evaluar cada factor de riesgo y tampoco se tiene un procedimiento que, en sus diferentes fases, logre combinar la gestión eficiente de riesgo y el desempeño laboral de los trabajadores, lo que es el aporte principal de la investigación.

\section{MATERIALES Y MÉTODOS}

La propuesta del procedimiento que permite valorar la incidencia de la gestión de los riesgos laborales en el desempeño laboral en usuarios de PVD consideró los métodos de investigación:

- Investigación exploratoria. Por cuanto se estudian los diferentes tipos de trabajadores que se encuentran laborando en la empresa y se realiza una selección de aquellos que son considerados como usuarios de PVD, haciendo la selección de para todo el proceso de investigación. La investigación exploratoria permitió, en la propuesta del procedimiento, la selección de aquellos métodos que posibilitan el diagnóstico de los riesgos laborales en usuarios de PVD.

- Método descriptivo. El método descriptivo permitió el reconocimiento de las características de las herramientas propuestas, la descripción de las características 
del trabajo con PVD y las posibles relaciones con el desempeño laboral. El método descriptivo fue usado en la primera etapa de la investigación, donde se describen las características sociodemográficas de los trabajadores seleccionados, y algunos elementos que permiten valorar el estado de salud y bienestar de sus trabajadores.

- Trabajo de campo. Se propone, en las etapas del procedimiento, un conjunto de herramientas y métodos para su aplicación en el lugar de trabajo, las cuales contribuyen a la obtención de los resultados de investigación.

- Análisis-síntesis. Para el diagnóstico de la situación actual en materia de prevención de riesgos laborales, se utilizó el método de investigación análisis-síntesis de la información recolectada mediante la técnica de la observación. El método análisis-síntesis se usa en el procesamiento de los resultados, la compilación de los datos obtenidos en cada herramienta.

\section{RESULTADOS}

La aplicación de los diferentes métodos de investigación en diferentes etapas contribuyó con la propuesta de las investigadoras acerca de un procedimiento que permitiera estudiar la incidencia de los riesgos laborales en el desempeño laboral, específicamente para aquellos usuarios de PVD. En la figura 1 se presenta el esquema del procedimiento propuesto. La investigación recoge dos variables importantes de estudio: la prevención de riesgos y el desempeño laboral de sus trabajadores, enfocando el estudio en aquellos empleados que son considerados como usuarios de PVD y que se encuentran expuestos a determinados factores de riesgo que afectan su desempeño, salud y bienestar.

\section{Etapa I. Diagnóstico inicial}

El diagnóstico inicial considera la identificación de aquellos trabajadores que, por las características de las actividades que realizan, deben ser considerados como usuarios de PVD. Una vez seleccionados, se le realiza un análisis de las condiciones de trabajo en su puesto de trabajo, considerando su entorno, los errores más frecuentes en sus tareas y los factores de riesgo a los que se exponen, mostrando sus consecuencias a corto, mediano o largo plazo.

\section{Trabajadores considerados como usuarios de PVD}

Para identificar a los usuarios de PVD, se toman como referencia los principales criterios emitidos en la guía técnica que desarrolla el Real Decreto n. 488 (1997) del Instituto Nacional de Seguridad y Salud en el Trabajo [INSST] en España, donde se definen los parámetros a considerar para identificar a los trabajadores como 


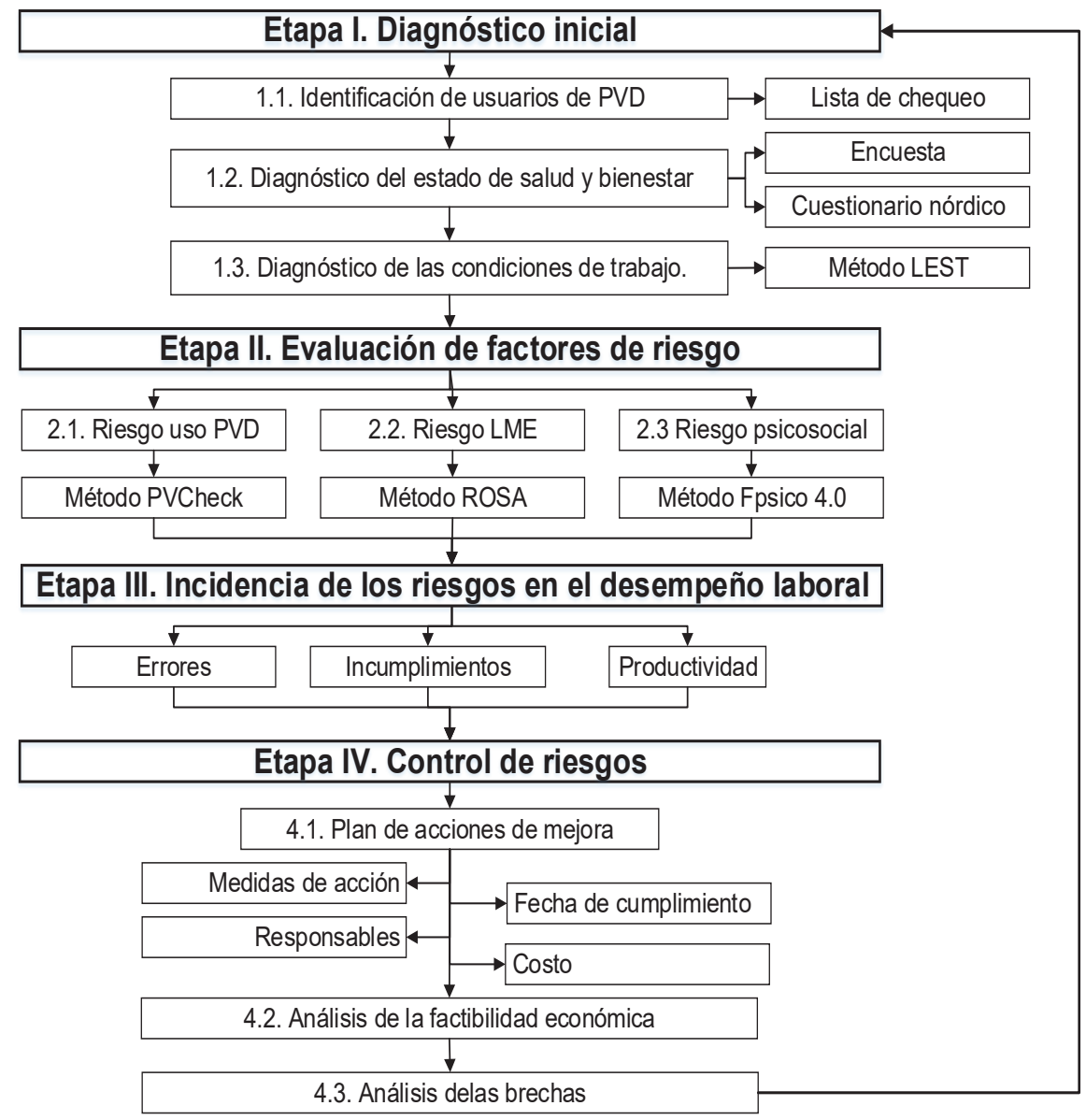

Figura 1. Procedimiento para el estudio de la prevención de riesgos laborales y su incidencia en el desempeño laboral

Elaboración propia

usuarios de PVD. Dichos parámetros, según el Instituto Nacional de Seguridad y Salud en el Trabajo (2006), son:

a) Los que pueden considerarse "trabajadores" usuarios de equipos con pantalla de visualización: todos aquellos que superen las cuatro horas diarias o veinte horas semanales de trabajo efectivo con dichos equipos.

b) Los que pueden considerarse excluidos de la consideración de "trabajadores" usuarios: todos aquellos cuyo trabajo efectivo con pantallas de visualización sea inferior a dos horas diarias o diez horas semanales. 
c) Los que, con ciertas condiciones, podrían ser considerados "trabajadores" usuarios: todos aquellos que realicen entre dos y cuatro horas diarias (o diez a veinte horas semanales) de trabajo efectivo con estos equipos.

La opción c) considera otros elementos que permiten determinar la cantidad de respuestas afirmativas (cinco respuestas de los siete criterios que se preguntan) para ser considerados usuarios de PVD. Su procesamiento se realiza en Excel y se presentan los resultados porcentuales por departamentos en la cual se identifican usuarios de PVD.

El resultado de esta etapa es la selección de los trabajadores con los cuales se continúa con el proceso de investigación en las siguientes fases, profundizando en el diagnóstico de su estado de salud y bienestar.

Diagnóstico del estado de salud y bienestar de los trabajadores

Las herramientas para el diagnóstico del estado de salud y bienestar aplicado a los usuarios de PVD permiten tener una visión de la situación actual en la que se encuentran los trabajadores, describiendo si han presentado malestares provocados por las condiciones que les impone el trabajo.

\section{Recomendaciones para la aplicación del cuestionario nórdico}

La herramienta fue aplicada al $100 \%$ de los trabajadores considerados como usuarios de PVD. El cuestionario nórdico fue creado por Kourina en 1987, mediante el análisis factorial que muestra la validez de constructo de la escala en versión española, que mantiene sus excelentes propiedades psicométricas. Este cuestionario arroja coeficientes de consistencia y fiabilidad entre 0,727 y 0,816 (Martínez et al., y otros, 2014). Estos resultados miden el grado en que una prueba aborda diferentes constructos y ofrece resultados fiables.

El cuestionario puede aplicarse usando las ventajas del uso de las tecnologías de la información y las comunicaciones. Una vez aplicado, se procede a su procesamiento y se exponen los resultados. Se recomienda el uso de gráficos que muestren las comparaciones entre los resultados obtenidos.

Diagnóstico de las condiciones de trabajo en los usuarios de PVD

Para el diagnóstico de las condiciones de trabajo en los usuarios de PVD, se propone el uso del método LEST, mediante el cual debe recogerse la información requerida para valorar cada una de las dimensiones que considera el método (ver tabla 1). 
Tabla 1

Resumen de las dimensiones y variables estudiadas en el método LEST

\begin{tabular}{lllll}
\hline Entorno físico & Carga física & Carga mental & Aspectos psicosociales & Tiempos de trabajo \\
\hline Ambiente & Carga estática & Apremio de & Iniciativa & Tiempo de trabajo \\
térmico & & Ciempo & \\
Ruido & Carga dinámica & Complejidad & Estatus social & \\
Iluminación & & Atención & Comunicaciones & \\
Vibraciones & & & Relación con el mando & \\
\hline
\end{tabular}

Fuente: Diego-Mas (2015)

En esta etapa, la aplicación de esta herramienta busca identificar posibles causas o acciones futuras de mejora en las subsiguientes etapas. Este método permite diferenciar en cuál de las cinco variables se tienen más dificultades.

La recolección de la información se realiza mediante una hoja de campo, en la que se detallan los elementos de la evaluación y del evaluador. En algunas de las dimensiones del método, como el entorno físico, es necesario el uso de diferentes equipos de medición que facilitan la evaluación de las variables, como es el caso de luxómetros para medir el nivel de iluminación, el sonómetro para la evaluación del ruido o el psicrómetro de aspiración para medir las temperaturas del aire y húmeda, entre otros. Para las variables de la carga física se utiliza el estudio de las operaciones en el proceso, usando técnicas como la representación de los diagramas de flujo, la grabación de actividades o el estudio de tiempo para valorar los tiempos de trabajo.

El procesamiento de la información se realiza con la ayuda de la aplicación informática que ofrece Ergonautas, en la cual se van colocando las valoraciones en cada una de las dimensiones que el método considera. Los resultados finales se valoran según la escala que se muestra en la tabla 2.

Tabla 2

Valoración de la puntuación final del método LEST

\begin{tabular}{ll}
\hline Puntuación & Valoración \\
\hline $0,1,2$ & Situación satisfactoria. \\
$3,4,5$ & $\begin{array}{l}\text { Débiles molestias. Algunas mejoras podrían aportar más comodidad al } \\
\text { trabajador. }\end{array}$ \\
6,7 & Molestias medias. Existe riesgo de fatiga. \\
8,9 & Molestias fuertes. Fatiga. \\
10 & Situación nociva. \\
\hline
\end{tabular}




\section{Etapa II. Evaluación de factores de riesgo}

En la fase 2 del procedimiento se tiene como objetivo realizar una evaluación de los factores de riesgo de una manera más detallada. A continuación, se presenta la propuesta de los métodos a utilizar.

\section{Aplicación del método PVCheck}

El método PVVCheck permite realizar una valoración detallada de las deficiencias encontradas en el entorno de trabajo y los usuarios de PVD. Para ello, se analizan cuatro dimensiones importantes:

- Parte I. Equipo de trabajo

- Parte II. Entorno de trabajo

- Parte III. Programas del ordenador

- Parte IV. Organización y gestión

Los pasos para la aplicación del método son:

1. Preparación del cuestionario. En este paso se procede a preparar el cuestionario y se adapta a las características de la empresa.

2. Identificación de grupos de trabajo homogéneos (GTH). Se identifica dentro de los usuarios de PVD a aquellos trabajadores que tienen las mismas características en cuanto al trabajo que realizan y los puestos asumidos.

3. Trabajo de campo. Se procede a aplicar el cuestionario en los GTH, tomando las evidencias de las deficiencias encontradas.

4. Procesamiento de la información. El procesamiento de la información se realiza mediante el software PVCheck en su versión 4.0, disponible en la página del INSST.

\section{Aplicación del método ROSA}

El método ROSA (Rapid Office Strain Assessment) fue publicado en 2011 por Sonne y otros colaboradores. Su objetivo es identificar las áreas de intervención prioritaria en el trabajo de oficina. En su evaluación, este método considera cinco elementos relacionados con el puesto de trabajo: silla, pantalla, teclado, mouse y teléfono.

El método de recolección de datos puede ser por observación directa o, preferentemente, por el estudio de la imagen en un video grabado. Son seleccionadas por el especialista las posturas más desfavorables y su duración se facilita por los usuarios de puestos PVD. 
En función de los datos obtenidos durante la observación de las posturas, se determinan dos posibles niveles de riesgo y de actuación. En la tabla 3, se muestran las valoraciones cualitativas y cuantitativas del método ROSA.

Tabla 3

Valoración del riesgo y niveles de actuación en el método ROSA

\begin{tabular}{llll}
\hline Puntuación & Riesgo & Nivel & Actuación \\
\hline 1 & Inapreciable & 0 & No es necesaria actuación. \\
$2-3-4$ & Mejorable & 1 & Pueden mejorarse algunos elementos del puesto. \\
5 & Alto & 2 & Es necesaria la actuación. \\
$6-7-8$ & Muy Alto & 3 & Es necesaria la actuación cuanto antes. \\
$9-10$ & Extremo & 4 & Es necesaria la actuación urgentemente. \\
\hline
\end{tabular}

Fuente: Diego-Mas (2015)

Aplicación del método Fpsico

La aplicación del método Fpsico, en su versión 4.0, permite realizar una evaluación de los factores de riesgo psicosocial a los que se encuentran expuestos los trabajadores bajo los siguientes pasos:

- Presentación y aprobación del cuestionario por la Alta Dirección

En esta parte se realiza una presentación a la Alta Dirección de las etapas a seguir con esta evaluación y se presenta el cuestionario.

- Reunión con todos los trabajadores

La reunión con los trabajadores se realiza agrupándolos en un espacio físico o virtual perteneciente a la empresa. En este encuentro se presentan los objetivos del estudio y se da el acceso al cuestionario. El uso de las herramientas digitales permite facilitar el acceso a la evaluación, pero es necesario garantizar el anonimato y la discreción en el proceso. Se explica que el cuestionario es anónimo y pueden responder con la verdad, debido a que los ayudará a mejorar las condiciones de trabajo.

\section{- Muestra de la población}

El tamaño de muestra se calcula para conocer el mínimo número de trabajadores que deben contestar el cuestionario para garantizar un nivel de confianza del $95 \%$ con un nivel de exactitud de $\pm 5 \%$, mediante la expresión: 


$$
n=\frac{z^{2} N p q}{e^{2}(N-1)+z^{2} p q}
$$

Donde:

n: número mínimo de personas que se necesita que respondan el cuestionario.

Z: estadígrafo de la normal. Se asume un nivel de confianza del $95 \%$ que asume un valor del estadígrafo de 1,96.

$\mathrm{N}$ : Población, cantidad de personas que forman parte de la empresa o área de estudio.

e: error absoluto, en este caso se considera el 5 \% (estudio conclusivo)

$p=q=$ probabilidad de éxito y fracaso. En este caso igual a 0,5

\section{- Aplicación y procesamiento de datos}

Una vez aplicados, los cuestionarios son recopilados y procesados. Para el procesamiento de la información se recomienda el uso del software Fpsico en su versión 4.0, que ofrece los resultados y gráficos finales. El método permite incorporar preguntas adicionales para realizar comparaciones.

\section{Etapa III. Incidencia de los riesgos laborales en el desempeño laboral}

Los usuarios de PVD pueden, en el desarrollo de sus actividades, ser afectados por las condiciones de trabajo a las que se exponen y que los hacen cometer errores que afectan el desempeño laboral y, con ello, la productividad.

Los métodos para evaluar el error humano parten de determinar el grado de incidencia de los errores cometidos en los procesos de interacción hombre-máquina en la industria manufacturera (Casares-Li, Rodríguez-Hernández y Viña-Brito, 2016, p. 65).

El error humano se define como una decisión o acción humana que produce - o tiene el potencial para producir - una reducción en la efectividad, salud y seguridad del trabajador, o una interrupción en el comportamiento del sistema sociotécnico, con un impacto inmediato o a largo plazo (Casares-Li et al., 2016, p. 3).

En esta parte del procedimiento se propone estudiar los fallos activos que se producen considerando el nivel 1 que se propone en la Tecnología de Tratamiento Ergonómico del Error Humano (TErEH) (Casares-Li et al., 2016, p. 4). Los indicadores a estudiar son:

1. Error. Dentro de los errores que pueden darse en el trabajo desarrollado por el personal usuario de PVD están los siguientes: 
- Omisión

- Sustitución

- Inserción

- Repetición

2. Incumplimientos. En este caso se violan los procedimientos o protocolo de trabajo establecidos para desarrollar sus actividades. Los tipos de violación consideradas son:

- Rutinaria

- Excepcional

- Necesaria

- Optimización

3. Productividad. El estudio de la productividad en esta investigación se enfoca a el trabajador usuario de PVD. En este caso se considera el porcentaje de aprovechamiento del tiempo de la jornada laboral y el estudio de las pérdidas de tiempo.

Las herramientas que permitirán diagnosticar estas variables son las recomendadas por los estudio y medición del trabajo, entre ellas:

- Fotografía individual detallada

- Fotografía colectiva detallada

La selección de la herramienta estará en función de las características del puesto específico a evaluar.

Serán considerados tres días de trabajo normales inicialmente, observando la jornada laboral completa, la cual permitirá clasificar los tiempos y determinar el número de días a observar, considerando un $95 \%$ de confianza y un error del $5 \%$.

\section{Etapa IV. Control de riesgos}

Plan de acciones de mejora

Las acciones de mejora se definen según la evaluación de riesgos realizada en las etapas anteriores. Estarán en función de los elementos que necesitan una mejora en el puesto que contribuya a la mejora de la seguridad, salud, bienestar y desempeño laboral de los trabajadores. Los elementos a definir en cada acción de mejora consideran: 
- Responsable

- Fecha de cumplimiento: inicio y fin

- Recursos necesarios

- Inversión

\section{Análisis de la factibilidad económica}

La factibilidad económica busca realizar un análisis de lo positivo en la aplicación en la empresa de las mejoras propuestas. Para ello, se propone el análisis de costo-beneficio.

Se analiza cuáles son las consecuencias de la no intervención, de la no mejora, de la no implementación de una gestión adecuada en la eliminación, mitigación o control de los riesgos en el área y se analiza la incidencia negativa para la salud, seguridad, bienestar y desempeño de los trabajadores ante situaciones adversas, estimando el costo de la no prevención y haciendo una comparación con la inversión propuesta para solucionar las limitantes encontradas para determinar si su aplicación es factible.

\section{Análisis de las brechas}

El análisis de las brechas permite determinar los aspectos que no se pueden cumplir en el momento de la intervención, se justifican las razones y se establecen como puntos que son necesarios darle continuidad en el mediano o largo plazo para contribuir a la mejora continua.

En este momento se determinan los porcentajes de cumplimiento de las acciones de mejora propuestas y se plantean las estrategias necesarias.

\section{DISCUSIÓN}

La integración de la prevención en la empresa es un concepto fundamental y es la primera obligación en materia de Prevención de Riesgos Laborales (García, 2018). La incidencia que tiene una buena gestión de riesgos laborales contribuye desde muchas aristas con la empresa y los trabajadores. Para la empresa, estos resultados se traducen en mayor rentabilidad, menores indicadores de siniestralidad y mayores ganancias, factores que no pueden garantizarse si el personal que brinda el servicio o realiza las actividades productivas tiene que someterse a constantes riesgos o situaciones que afectan su seguridad, salud y bienestar.

El desempeño laboral tiene una relación directa con las condiciones laborales a las que se exponen los trabajadores y el bienestar que tengan en su puesto de trabajo. El talento humano hace que las organizaciones sean productivas y competitivas, por lo cual 
se hace ineludible el compromiso del trabajador con su organización, desvirtuando cualquier conflicto que deteriore su estadía en la empresa (Ccollana-Salazar, 2017).

Algunos autores han desarrollado investigaciones en el campo de la prevención de riesgos laborales y su contribución al mejor desempeño laboral. Entre ellas, la publicada por Real et al. (2019) donde se estudia el Régimen de Trabajo y Descanso (RTD) en una empresa hotelera y su incidencia en el incremento de la productividad laboral, que ayuda a valorar y proponer mejoras que permiten el incremento del bienestar laboral y un mayor rendimiento. En esta investigación se evidencia que el considerar el estudio del tiempo de trabajo y el descanso es una variable que permite el diseño de actividades más eficientes y productivas. En este caso, en las tres áreas de estudio hotelero se muestra la propuesta de los tiempos totales de descanso (Real et al., 2019).

Otros autores han enfocado sus estudios en un procedimiento diseñado y desarrollado para empresas de manufactura y de servicios que permite optimizar el desempeño en los sistemas de trabajo. En esta propuesta, se muestran en dos fases y cinco etapas las acciones que se deben desarrollar para la implementación de acciones de mejoras ergonómicas en los puestos (Mejías, 2018). Sin embargo, otros identifican los factores determinantes de la productividad laboral en las pequeñas y medianas empresas (pymes) del sector Confecciones del área metropolitana de Bucaramanga en Colombia, aplicando una encuesta sobre productividad laboral desde dos dimensiones: la humana y la del proceso productivo (Jaimes, Luzardo y Rojas, 2018) y otras investigaciones como la de Hernández y Morales (2017) identifican los factores que determinan la motivación y satisfacción laboral que impactan positivamente en la eficacia y eficiencia de los empleados públicos en un organismo autónomo de la administración pública del Estado de Sinaloa.

En el siglo XXI la mayoría de los puestos de trabajo necesita el uso de PVD, por lo que es responsabilidad del empleador garantizar condiciones de trabajo que no afecten el desempeño laboral con el único de fin de alcanzar alta competencia en el mercado (Carmona y Rosas, 2017).

La propuesta del procedimiento es una herramienta útil para que todos aquellos encargados de la prevención de riesgos laborales y el manejo del talento humano en las organizaciones puedan gestionar adecuadamente los riesgos en los puestos con usuarios de PVD y su incidencia en el desempeño laboral. La selección y facilidad de las herramientas propuestas en cada una de las etapas del procedimiento permite la gestión de riesgos de una manera sencilla, que garantice un trabajo digno y que permita el desempeño adecuado sin afectar a los trabajadores ni a las organizaciones. 


\section{CONCLUSIONES}

La prevención de riesgos laborales toma cada vez mayor importancia para garantizar altos índices de competitividad, productividad y eficiencia en el desempeño laboral en las organizaciones, por lo que considerar dentro de los procesos de gestión estos lineamientos es un factor esencial.

El procedimiento puede ser aplicado al trabajo de oficina, ya sea en las áreas de trabajo habituales o en las condiciones actuales, es decir, en los espacios donde se desarrolla el teletrabajo. Dentro de las limitaciones que tiene el procedimiento está que no puede llevarse a cabo si la Alta Gerencia y los trabajadores no asumen el compromiso de colaborar en la investigación. El consentimiento de los trabajadores es fundamental para detectar las brechas y poder implementar acciones de mejora acorde a las situaciones reales de cada organización.

La propuesta del procedimiento, con sus cuatro etapas, analiza y estudia un conjunto de herramientas y métodos que permiten el cumplimiento de su objetivo, pudiendo realizar una evaluación de los factores de riesgo laboral y su incidencia en el desempeño laboral en usuarios de PVD.

La primera etapa se enfoca en realizar el diagnóstico inicial, considerando cuántos trabajadores dentro de la empresa pueden ser usuarios de PVD y se realiza un diagnóstico del estado de salud y el bienestar en su puesto, para ello se propone una encuesta que permita recopilar los datos del trabajador y el cuestionario nórdico para valorar los síntomas y malestares de los trabajadores durante el desarrollo de sus actividades. Los resultados de esta etapa permiten a las organizaciones mapear su situación actual y tomar las decisiones adecuadas en continuar el resto de las fases. En caso que en una organización en esta etapa no se detecten signos alarmantes, se detiene el proceso y la observación debe continuar de manera periódica.

En la segunda etapa, considerando aquellos puestos en los que se han detectado problemáticas, se realiza la evaluación detallada, aplicando el método PVcheck, el método ROSA y el método Fpsico, que permiten valorar los factores de riesgos por el uso de PVD, el riesgo de LME y los factores de riesgo psicosocial.

La tercera etapa permite el análisis de la incidencia que han tenido estos riesgos en el desempeño laboral de los trabajadores; para ello, se propone el estudio de los errores que se comenten en sus actividades laborales, se analizan los incumplimientos y se calcula la productividad en el trabajo. Estos resultados permiten demostrar que el desempeño laboral se encuentra muy influenciado por las condiciones en las cuales se realiza la actividad laboral.

El impacto de estas variables y el aporte del procedimiento propuesto permitirá que realizar las adecuaciones a los puestos de trabajo y se vuelva a evaluar el 
desempeño laboral, mediante indicadores adaptados a las características del puesto evaluado.

El análisis del costo-beneficio en cada una de las acciones de mejora permite demostrar a la Alta Dirección las ventajas en la gestión de riesgos y la incidencia en el desempeño laboral.

\section{REFERENCIAS}

Arboleda Posada, G. I. y Cardona Jiménez, J. L. (2018). Percepción de la satisfacción con la labor desempeñada y factores de motivación del personal de las instituciones prestadoras de servicios de salud (IPS) del valle de Aburrá. Gerencia y Políticas de Salud, 17(35). https://doi.org/10.11144/Javeriana.rgsp17-35.psld

Asamblea Nacional República del Ecuador (2008). Constitución de la República del Ecuador. Publicada en el Registro Oficial n. ${ }^{\circ}$ 449, 20 de octubre del 2008. Recuperado de https://www.google.com/url?sa=t\&rct=j\&q=\&esrc=s\&source= web\&cd=\&ved=2ahUKEwjU-06ZjpfrAhUvrFkKHZxpCeEQFjAEegQIERAB\&url=ht tps\%3A\%2F\%2Fwww.asambleanacional.gob.ec\%2Fsites\%2Fdefault\%2Ffiles\%2 Fdocuments\%2Fold\%2Fconstitucion_de_bolsillo.pdf\&usg=AOvVaw2x-HTv8XnVGWgIFpYI3dh5

Aulestiarte, A. y Villanueva, P. (2013). Análisis comparativo de métodos ergonómicos. Aplicación de casos. Escuela Técnica Superior de Ingenieros Industriales y de Telecomunicación. Recuperado de https://academica-e.unavarra.es/handle/2454 17769

Bravo, M. E. y Salazar, J. C. (2017). El clima laboral y su incidencia en el desempeño del personal docente de una escuela de educación básica en Ecuador. PODIUM, $131-143$.

Buitrago, A. (2016). Utilidad de las metodologías REBA, RULA y OCRA para valorar la carga física en trabajadores de una empresa del sector floricultor. Universidad Nacional de Colombia. Recuperado de http: //bdigital.unal.edu.co/55012/1/65829162.2016.pdf

Carballé Piñón, R. de los Á. (2015). Estrategia para elevar la motivación laboral; factor imprescindible para mejorar nuestra productividad. InfoCiencia, 19(4), 1-12.

Carmona-Fuentes, P. y Rosas-Reyes, R. E. (2017). Influencia de la inteligencia emocional en el desempeño laboral. Líder: Revista Labor Interdisciplinaria de Desarrollo Regional, 19(31), 107-118.

Casares-Li, R., Rodríguez-Hernández, A. G. y Viña-Brito, S. J. (2016). Análisis de errores humanos mediante la tecnología TErEH: experiencias en su aplicación. Ingeniería Industrial, 37(1), 49-58. 
Ccollana-Salazar, Y. (2017). Rotación del personal, absentismo laboral y productividad de los trabajadores. San Martín Emprendedor, 6(2), 40-49.

Cuesta-Santos, A. y Valencia-Rodríguez, M. (2018). Capital humano: contexto de su gestión. Desafíos para Cuba. Human Capital: Context of their management. Challenges for Cuba, 39(2), 135-145.

Diego-Mas, J. A. (2015). Análisis ergonómico global mediante el método LEST. Ergonautas, Universidad Politécnica de Valencia. Recuperado de http://www.ergonautas.upv. es/metodos/lest/lest-ayuda.php

Diego-Mas, J. A. (2015). Evaluación de puestos de trabajo de oficinas mediante el método ROSA. Ergonautas, Universidad Politécnica de Valencia. Recuperado de http:// www.ergonautas.upv.es/metodos/rosa/rosa-ayuda.php

Durany Puig, 0. (2019). PRL Industria cárnica. Movimientos repetitivos. Gestión Práctica de Riesgos Laborales, 168, 16-20.

García, R. F. (2018) Prevención de riesgos laborales. Derechos y deberes de los participantes. Sus ventajas para las empresas. Gestión práctica de riesgos laborales: integración y desarrollo de la gestión de la prevención, 159, 8-21.

Hernández Juárez, J. L. y Morales Morales, J. R. (2017). Evaluación de la motivación y satisfacción laboral en un organismo autónomo de la administración pública del Estado de Sinaloa. Revista Daena (International Journal of Good Conscience), 12(2), 107-147.

Instituto Nacional de Seguridad y Salud en el Trabajo [INSST] (2006). Evaluación y prevención de los riesgos relativos a la utilización de equipos con pantallas de visualización. Recuperado de https://www.insst.es

Jaimes, L.; Luzardo, M. y Rojas, M. D. (2018). Factores determinantes de la productividad laboral en pequeñas y medianas empresas de confecciones del área metropolitana de Bucaramanga, Colombia. Información Tecnológica, 29(5), 175-186. https:// doi.org/10.4067/S0718-07642018000500175

López, J. A. S.; Camal, M. del C. G. y Galicia, W. A. M (2018). Evaluación del desempeño laboral y su incidencia en la productividad en las tortillerías de la localidad de escárcega. Revista Daena (International Journal of Good Conscience), 13(1), 317-327.

Martínez, B.; Santo Domingo, S.; Bolea, M.; Casalod, Y. y Andrés, E. (2014). Validación del cuestionario nórdico musculoesquelético estandarizado en población española. Prevención integral. Recuperado de https://www.prevencionintegral.com/ca/ canal-orp/papers/orp-2014/validacion-cuestionario-nordico-musculoesqueletico-estandarizado-en-poblacion-espanola 
Mejías-Herrera, S. H. (2018). Las herramientas de intervención ergonómica: consideraciones conceptuales y experiencias prácticas en Cuba y Brasil, Ingeniería Industrial, 39(1), 3-14.

Palacio, E. V. G.; Estrada, G. J. A.; Restrepo, K. D. y Toro, S. G. (2017). Relación entre los desórdenes musculo-esqueléticos y la postura frente a una pantalla de visualización de datos. VIREF Revista de Educación Física, 6(1), 1-12.

Paredes Rizo, M. L. y Vásquez Ubago, M. (2018). Estudio descriptivo sobre las condiciones de trabajo y los trastornos musculo esqueléticos en el personal de enfermería (enfermeras y AAEE) de la Unidad de Cuidados Intensivos Pediátricos y Neonatales en el Hospital Clínico Universitario de Valladolid. Medicina y Seguridad del Trabajo, 64(251), 161-199.

Real, G. L. R.; Marrero, N. H.; Lezcano, M. D. R. y Ávila, A. A. H. (2019). Valoración de los regímenes de trabajo y descanso. Caso de estudio: Varadero (Cuba). Anuario Turismo y Sociedad, 24, 149-160. doi: https://doi.org/10.18601/01207555.n24.07

Real Decreto n. ${ }^{\circ} 88$ (14 de abril de 1997) Disposiciones mínimas de seguridad y salud relativas al trabajo con equipos que incluyen pantallas de visualización. Recuperado de https://infantilprl.saludlaboral.org/wp-content/uploads/2017/01/488_pvd.pdf

Sonne, M.; Villalta, D. L. y Andrews, D. M. (2011). Development and evaluation of an office ergonomic risk checklist: ROSA-Rapid office strain assessment. Applied ergonomics, 43(1), 98-108. 